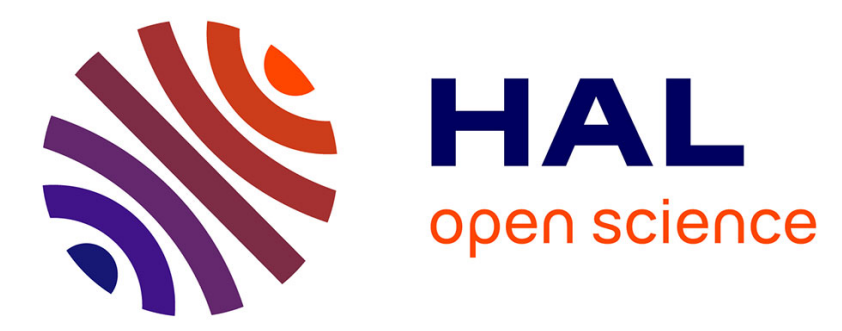

\title{
Les épreuves de la fidélité. La trahison dans les Sophonisbe sur les scènes italienne, française et anglaise aux XVIe et XVIIe siècles
}

Clotilde Thouret

\section{- To cite this version:}

Clotilde Thouret. Les épreuves de la fidélité. La trahison dans les Sophonisbe sur les scènes italienne, française et anglaise aux XVIe et XVIIe siècles. Seizième siècle, 2009, La trahison, 5, pp.93-114. hal-02902959

\section{HAL Id: hal-02902959 \\ https://hal.univ-lorraine.fr/hal-02902959}

Submitted on 20 Jul 2020

HAL is a multi-disciplinary open access archive for the deposit and dissemination of scientific research documents, whether they are published or not. The documents may come from teaching and research institutions in France or abroad, or from public or private research centers.
L'archive ouverte pluridisciplinaire HAL, est destinée au dépôt et à la diffusion de documents scientifiques de niveau recherche, publiés ou non, émanant des établissements d'enseignement et de recherche français ou étrangers, des laboratoires publics ou privés. 


\section{LES EPREUVES DE LA FIDELITE. LA TRAHISON DANS LES SOPHONISBE SUR LES SCENES ITALIENNE, FRANÇAISE ET ANGLAISE AUX XVI ${ }^{\mathrm{E}}$ ET XVII ${ }^{\mathrm{E}}$ SIECLES}

\section{Multiplicité des trahisons et conflits des fidélités}

S'intéresser aux Sophonisbe du théâtre des XVI et XVII e siècles pour tenter de « tirer la Trahison de sa fosse obscure' » peut sembler quelque peu paradoxal, ou du moins inattendu. La petite-fille d'Hamilcar est en effet plutôt connue, ou convoquée, comme «femme illustre » ou bien comme héroïne de tragédie élégiaque². Qu'elle soit louée pour son courage et sa fermeté au moment de boire le poison qui lui évitera le triomphe, ou que l'on pleure sur son impossible union avec Massinisse, roi Numide et allié des Romains, on ne voit guère où pourrait se loger le traître. Pourtant, l'histoire de Sophonisbe, racontée en particulier par Tite-Live et Appien d'Alexandrie dans leurs histoires romaines, peut se lire comme une succession de trahisons, effectuées ou refusées.

Avant de rappeler les principaux éléments de cet épisode de la seconde guerre punique, il convient de préciser que ces deux historiens sont les sources principales de tous les poètes qui ont consacré des pages à l'héroïne carthaginoise, même si la source antique se trouve ponctuellement contaminée par une réécriture renaissante ou baroque . Les aventures de Massinisse et de Sophonisbe se trouvent donc au Livre XXIX (19-33) et au Livre XXX (3-15) de l'Histoire romaine de Tite-Live ; chez Appien, elles se distribuent entre L'Ibérique (Histoire romaine, Livre VI, ch. 37 en particulier) et Le Livre Africain qui narre la campagne de Scipion en Afrique (Histoire romaine, Livre VIII, ch. 10-27).

\footnotetext{
'L'expression est celle de JUSTE LIPSE (Les Politiques. Livre IV, 1597, éd. Jacqueline Lagrée, Caen, Presses Universitaires de Caen, 1994, p. 111).

"Voir Christian DELMAS, "Les Sophonisbe et le renouveau de la tragédie en France », XVII siècle, 2000, n²08, p. 443-464; Bénédicte LOUVAT, «Introduction», in Jean Mairet, Théâtre complet, t. I, Paris, Champion, 2004, p. 27-97.

${ }^{3} \mathrm{D}^{\prime}$ autres historiens antiques ont raconté cet épisode, Polybe, Diodore de Sicile ou Dion Cassius par exemple. Les principales versions ou réécritures de l'histoire de Sophonisbe et de Massinisse, en Europe et jusqu'à la fin du XVII siècle, tous genres confondus, sont les suivantes (ce sont les éditions auxquelles je ferai référence) : PetrarQue, L'Afrique. Affrica, I-V, éd. et trad. Pierre Laurens, Paris, Les Belles Lettres, 2006, livre V ; BOCCACE, De Claris Mulieribus, 1362, Des Dames de renom. Nouvellement traduit en françois d'après la traduction de L. A. Ridolfi, Lyon, Guillaume Rouillé, 1551, éd. Xavier Carrère, Toulouse, Editions Ombres, 1996 ; Galeotto DEL CARRETTO, Sofonisba, 1502 ; Gian Giorgio TRISSINO, Sofonisba, 1514, dans Il teatro italiano II. La tragedia del Cinquecento, t. 1, éd. Marco Ariani, Turin, Einaudi, 1977 ; Mellin de SAINT-GELAIS, Sophonisba. Tragédie très excellente tant pour l'argument que pour le poli langage et graves sentences dont elle est ornée, représentée et prononcée devant le Roi, en sa ville de Blois, Paris, Gilles Corrozet, 1559, éd. Luigia Zilli, dans La Tragédie à l'époque d'Henri II et de Charles IX, Première série, vol. 1 (1550-1561), Florence-Paris, Leo S. Olschki-Presses Universitaires de France, 1989, p. 238-368; BANDELlO, Novelle, I, XLI, 1554 ; Nicolas de MonTREUX, La Sophonisbe, Rouen, Raphaël du Petit Val, 1601, éd. Donald Stone, Jr., Genève, Droz, 1976 ; Antoine de MONTCHRESTIEN, La Carthaginoise, ou la Liberté, 1604, dans Les Tragédies de Montchrestien, éd. L. Petit de Julleville, Paris, Plon, 1891 ; John MARSTON, The Wonder of Women, or the Tragedie of Sophonisba, 1606, La tragédie de Sophonisbe, ou la Merveille des femmes, éd. et trad. Jacqueline Pelorson, Paris, Les Belles Lettres, 1996 ; Jean MAIRET, La Sophonisbe, 1634, dans Théâtre du XVII siècle, t. I, éd. Jacques Scherer, Paris, Gallimard, Bibliothèque de la Pléiade, 1975 ; CORNEILlE, Sophonisbe, 1663, dans CEuvres complètes, t. III, éd. Georges Couton, Paris, Gallimard, Bibiothèque de la Pléiade, 1987 ; Madeleine de SCUDERY, Les Femmes illustres, 1644, éd. Claude Maignien, Paris, Côté-femmes, 1991 ; Nathaniel LEE, Sophonisba, or Hannibal's Overthrow, dans The Works of Nathaniel Lee, vol. 1, éd. Thomas B. Stroup et Arthur L. Cooke, New Brunswick, The Scarecrow Press, 1954. Sur les différentes versions, voir C. RICCI, Sophonisbe dans la tragédie classique italienne et française, Turin, Paravia, 1904, et A. AXELRAD, Le thème de Sophonisbe dans les principales tragédies de la littérature occidentale (France, Angleterre, Allemagne), Lille, Bibliothèque universitaire, 1956.
} 
Les réécritures des XVI et XVII siècles se concentrent sur la fin de l'épisode, proprement tragique. Sophonisbe, fille d'Hasdrubal, a été mariée à Syphax, roi de Numidie. À la suite de ce mariage, ce dernier est passé dans le camp carthaginois alors qu'il avait été allié des Romains pendant longtemps. Après ses victoires en Espagne, Scipion décide de porter la guerre en Afrique et il s'appuie pour cela sur un autre roi Numide, Massinisse. Dans certaines versions, Syphax a usurpé le trône de Massinisse. S'ils sont tous deux Numides, l'un (Massinisse) est le chef des Massyles et l'autre (Syphax) des Masaesyles, deux peuples qui se partagent une partie du territoire de l'actuelle Afrique du Nord. Après plusieurs batailles, les armées romaines de Scipion et Lélius et celles de Massinisse mettent en échec Syphax à Cirta (actuelle Constantine), capitale de ses États, et le font prisonnier. Alors que les soldats entrent dans la ville, Sophonisbe fait promettre à Massinisse de ne pas la livrer comme prisonnière aux Romains; il excède sa demande et l'épouse le jour même. Mis en garde par Syphax contre la belle Carthaginoise, rendue responsable de sa trahison, Scipion reproche alors à Massinisse d'oublier leur alliance et réclame Sophonisbe, qui appartient à Rome comme le reste du butin. Pour honorer sa promesse à la reine, le Numide lui donne (ou lui fait envoyer) du poison, qu'elle boit sans hésiter.

Dans la perspective qui nous intéresse ici, on voit qu'une telle histoire met en jeu deux grands moments de trahisons, réelles ou virtuelles, deux moments où s'éprouvent la foi et la parole donnée. Le premier est celui de la préparation de la campagne africaine de Scipion, le second, celui de la captivité de Sophonisbe. Alors que le premier déploie tout un enchaînement de traîtrises, le deuxième met en scène les contradictions de la fidélité, ou plutôt la contradiction des fidélités.

Syphax a joué un rôle important dans la seconde guerre punique parce qu'il était un roi puissant et parce qu'il a orchestré et accueilli des négociations de paix entre les deux camps. Il est d'abord l'ami des Romains, mais les Carthaginois lui donnent Sophonisbe dont il était profondément amoureux, et gagnent ainsi un allié. Selon Appien, la jeune fille avait été promise par Hasdrubal à Massinisse ; aussi ce dernier fait-il défection pour faire alliance avec Scipion. Cette donnée, on le verra, peut être décisive dans le deuxième moment de l'histoire. Constatons pour l'instant que la position intermédiaire des Numides favorise la multiplication des retournements $\mathrm{d}$ 'alliances et des trahisons: les Carthaginois trahissent Massinisse qui se bat à leurs côtés ; leur décision provoque la trahison des Romains par Syphax et donc celle de Carthage par Massinisse qui passe d'un camp à l'autre ; ce dernier, une fois allié à Scipion, est amené à se battre contre son propre peuple puisqu'il doit affronter Syphax; par ailleurs, l'alliance d'un Numide avec les Romains peut en soi s'interpréter comme une trahison puisque son allié naturel devrait être Carthage.

Une fois acquise la victoire contre Syphax, les termes dans lesquels la question de la trahison se pose ne sont plus seulement militaires. Aux allégeances politiques viennent s'ajouter les promesses privées ou conjugales: les fiançailles brisées qu'il faut renouer, la promesse faite à Sophonisbe de lui éviter la captivité romaine, le serment sacré du mariage. Or, du point de vue de Massinisse, la fidélité à ces serments entre en contradiction avec la fidélité qu'il doit aux Romains. C'est ce

\footnotetext{
' Le récit d'APPIEN est très clair (Histoire romaine. Livre VI. L'Ibérique, éd. et trad. Paul Goukowsky, CUF, 2003, XXXVII-149-150) : "À l'insu d'Asdrubal, Massinissa franchit de son côté le détroit et, après avoir établi des liens d'amitié avec Scipion, il jura d'être son allié, s'il faisait une expédition en Afrique. Il avait conclu cet accord, en homme d'une fidélité à toute épreuve, pour la raison que voici. La fille d'Asdrubal (le général dont Massinisse était alors le compagnon d'armes) lui avait été promise en mariage. Mais le prince Syphax brûlait d'amour pour cette fille et les Carthaginois, qui jugeaient capital de s'assurer de son appui contre les Romains, lui donnèrent la fille sans s'être informés de rien auprès d'Asdrubal. Quand la chose fut accomplie, Asdrubal s'efforça de la tenir secrète par égard pour Massinissa. Mais celui-ci l'apprit et conclut un accord avec Scipion. »
} 
conflit des fois jurées qui conduit à la mort de Sophonisbe ; la mort de la reine permet en effet à Massinisse de respecter à la fois la promesse qu'il lui a faite et son amitié avec les Romains. Sophonisbe quant à elle reste libre et fidèle à sa patrie comme à la dignitié royales. La spirale des trahisons s'achève donc sur des exemples de fidélité.

L'articulation des fidélités (militaire, amicale, politique, publique, privée, conjugale...) et les modalités de leur mise en conflit sont différentes d'une pièce à l'autre, mais l'on peut dès à présent faire trois ensembles de remarques :

(1) Tout d'abord, dans les différentes versions considérées, «trahison » possède le sens large que l'on trouve dans les dictionnaires du temps, celui de «Perfidie, défaut de fidélité à son Prince, à son ami, à celui qui avait de la confiance en une personne »(Furetière) 6 . Si «trahir» (ou «betray ») acquiert une forte connotation militaire ou si son sens se spécialise en "livrer à l'ennemi », notamment par l'influence des deux grandes figures de traîtres que sont Ganelon et Judas, le verbe désigne d'abord le fait d'être déloyal, de tromper la confiance d'un groupe ou d'une personne, de ne pas respecter son serment.

(2) C'est justement pour cette raison que les réécritures dramatiques de l'histoire de Massinisse et Sophonisbe peuvent retenir notre attention : les allégeances, les pactes, les serments se multiplient et se superposent au point de se contredire et de placer des personnages dans des impasses, de les écarteler entre deux fois jurées. Ainsi certains personnages se trouvent placés dans des situations particulièrement intéressantes puisqu'ils sont à la fois fidèles et parjures, loyaux et traîtres. Considérons par exemple le cas de Sophonisbe. Contrainte par le Sénat de Carthage, elle trahit la foi qu'elle a donnée à Massinisse ; une fois mariée à Syphax, elle trahit le peuple de Numidie en engageant Syphax à mener une guerre dans laquelle il a tout à perdre ; enfin, elle trahit le serment conjugal qui l'unit à Syphax. Cependant, tout au long de ces événements, elle reste fidèle à Carthage ; en se mariant à Massinisse, elle peut revendiquer une fidélité à la parole donnée par Hasdrubal et par ellemême au roi Numide; plus généralement, pour reprendre les mots de Bénédicte Louvat, Sophonisbe est une reine qui pratique "l'infidélité conjugale au nom de la fidélité politique ${ }^{7} »$. Quant à Syphax, il trahit l'amitié des Romains, mais il est en cela fidèle à son union avec Sophonisbe. J'ai déjà évoqué la situation de Massinisse et l'analyse des pièces me donnera l'occasion d'y revenir.

(3) En recentrant l'action sur la prise de Cirta et ses conséquences, les dramaturges écartent les motifs, pourtant particulièrement dramatiques, de la dissimulation du traître qui ourdit son crime (Iago-Othello) ou de la révélation du forfait (Auguste-Cinna)s. Nos textes présentent donc en quelque sorte des trahisons sans mensonge, ou sans duplicité. Si les pièces y perdent

\footnotetext{
- Voir par exemple, chez PETRARQuE, le message que Massinisse transmet à la reine par l'intermédiaire de l'esclave chargé du poison : op. cit., p. 184 : «Va, dit-il, cet affreux présent porte à la triste / Reine, me délivrant du serment fait aux dieux. / Qu'elle sache du moins que je tiens mes promesses. / Je remplis la seconde en attestant les dieux / Que l'autre m'agréait et qu'en vain j'essayai / De mon mieux d'obtenir qu'elle fût une reine / Et une épouse heureuse. »

'En anglais, en son plus ancien et premier sens, «treason » veut dire « the action of betraying ; betrayal of the trust undertaken by or reposed in any one; breach of faith » (Oxford English Dictionary). Le mot de «trahir » est passé en français avec le sens de «manquer à la foi donnée à quelqu'un »; puis au XVI siècle, le verbe prend d'autres acceptions : " ne pas respecter (un pacte) », « abandonner en passant à l'ennemi » (Dictionnaire historique de la langue française).

B. LOUVAT, op. cit., p. 55.

- Il faut signaler dès maintenant que la pièce de MARSTON fait exception puisqu'elle met en scène la trahison de Massinisse par le Sénat carthaginois, alors qu'il se bat encore contre les Romains, et l'échec de sa tentative d'assassinat. J'y reviendrai plus loin.
} 
en suspense ou en intensité dramatique, elles gagnent en revanche un espace pour le débat sur la trahison et sa légitimité, sur les fidélités et leur hiérarchisation, sur les manières de résoudre les conflits entre des loyautés qui sont en concurrence. Pour toutes ces raisons, le sujet de Sophonisbe se prête plus qu'un autre pour poser la question suivante : à qui (ou à quoi) est-ce que l'on se doit?

On peut faire l'hypothèse qu'il y a là une des raisons du succès de ce sujet au théâtre, à la fin de la Renaissance et à l'âge baroque. Dans son article consacré aux Sophonisbe, Christian Delmas explique de deux manières le rôle de ce sujet dans le renouveau de la tragédie et son prestige persistant. D'une part, il s'agit d'un sujet tiré de l'histoire romaine et qui met en scène un retournement de fortune éclatant. D'autre part, le personnage de Massinisse ouvre une perspective élégiaque et se prête au motif du vainqueur vaincu; quant à Sophonisbe, sa situation est propice aux déplorations pathétiques et "concentre sur sa personne la figure de l'oxymore" » puisqu'elle est mariée à l'instant de ses funérailles (l'idée est déjà chez Tite-Live). Il me semble qu'il faut ajouter à ces raisons la capacité de ce sujet à s'ouvrir à une réflexion sur la trahison, question qui possédait alors une acuité toute particulière. La contradiction des fidélités, ou le paradoxe du reniement qui permet de conserver sa foi, rencontre en effet des préoccupations nées d'un contexte historique particulier, celui de l'ébranlement de l'ordre féodal - qui se trouve être précisément fondé sur les liens de fidélité - par la progression du pouvoir royal et de l'État monarchique, et celui des guerres civiles (ou de leur menace) où la division des partis oblige souvent à devenir infidèle.

La question de la fidélité se trouve nécessairement posée dans les pièces qui représentent le sort tragique de l'héroïne carthaginoise, mais elle ne l'est pas toujours avec la même insistance ou avec la même intensité. Trissino, Montchrestien et Mairet n'en font pas un thème central, alors que chez Montreux, Marston et Corneille, le reniement et la fidélité, leur nature et leur objet, ordonnent l'ensemble de l'action et des discours.

\section{Les tragédies de Trissino, Montchrestien et Mairet: la promesse comme approfondissement du tragique}

Chez Trissino, Montchrestien et Mairet, le conflit des fidélités relève avant tout d'une logique dramaturgique. Il est l'instrument principal de la construction de l'impasse tragique dans laquelle se trouvent les personnages et vient éventuellement rehausser la lyrique amoureuse.

La pièce de Trissino, composée en 1514 et publiée en 1529 à Vicence, se donne pour la première tragédie régulière italienne. Elle est représentée pour la première fois à la cour de France en 1556, après avoir été traduite par Mellin de Saint-Gelais à la demande de Catherine de Médicis. L'Italien contamine les deux sources antiques et centre la tension tragique autour du drame intérieur de Sophonisbe, figure de la constance et de la vertu malheureuse malgré le topique renversement de fortune. Fidèle à Tite-Live, Boccace invoquait la luxure du Numide pour expliquer cette union au milieu du pillage de la ville, à laquelle il donnait d'ailleurs un caractère assez sulfureux ${ }^{10}$. Trissino choisit au contraire de prendre la suite de Pétrarque en

\footnotetext{
C. DELMAS, op. cit., p. 448.

${ }^{10}$ BOCCACE, op. cit., p. 70 : «Massinissa, qui même était Numide, et adonné (comme ils sont tous aussi) à luxure, regardant la douceur du visage de Sophonisbe (car son propre malheur la favorisait encore de certaine grâce, outre l'accoutumée, digne de compassion), en partie ému de telles prières attrayantes, et en partie surpris d'amour, tout ainsi armé qu'il était (n'étant pas encore arrivé Laelius) lui tendit la main droite, en gage de promesse et foi de ce qu'elle lui avait demandé, la levant debout ; et parmi les pleurs et plaintes des femmes, et entre le tumulte des soldats, qui rôdaient partout, incontinent la prit à femme, célébrant ses noces au milieu de la tempête des Romains, sous opinion
} 
faisant de Massinisse un chevalier courtois, vertueux et sensible. Sa promesse est ainsi qualifiée de "courtoise » et "magnanime »". Le recours à Appien va dans ce sens puisque l'on voit Massinisse aux actes III et IV rappeler aux Romains qu'il ne fait que reprendre ce qui lui a été volé et que son mariage n'est pas le mouvement d'un appétit désordonné mais un effet de sa pitié.

Cependant la pièce ne fait pas de la trahison de la foi donnée un principe structurant du texte. Aucun des chœurs n'y fait allusion. Le débat entre Scipion et Massinisse, qui occupe toute une partie de l'acte IV, porte avant tout sur ce qui fait la légitimité d'un mariage et sur ses droits à revendiquer Sophonisbe. Ensuite, Massinisse se rend très vite aux raisons du général romain :

Ben io vi priego assai che non vi spaccia

s'io cerco aver rispetto a la mia fede,

la qual troppo obligai senza pensarvi ; $[\ldots]^{\text {n. }}$.

Après quelques soupirs et sanglots, il fait envoyer le poison avec ces mots (c'est le serviteur qui parle) :

\footnotetext{
Madonna, il mio signore a voi mi manda,

e dice che servato volentieri

v'aría la prima sua promessa fede,

sí come devea far marito a moglie ;

ma poi che questo da la forza altrui

gli è tolto, ecco vi serva la seconda :

che non andrete viva ne le forze

d'alcun romano, e però vi ricorda

di far cosa condegna al vostro sangue ${ }^{\text {. }}$.
}

Au début du dernier acte, il envisagera bien de faire enlever Sophonisbe pour l'emmener à Carthage, mais c'est là un mouvement d'amour et de pitié et la reine a déjà commencé à ressentir les effets du poison. La solution est donc vite trouvée qui préserve la fidélité de Massinisse et c'est le personnage de Syphax qui cristallise sur lui toute la trahison (traître envers les Romains, mais aussi envers Sophonisbe, image de "parfaite beauté, douceur, courtoisie et bonté », qu'il rend seule responsable du retournement $\mathrm{d}^{\prime}$ alliance $\left.{ }^{14}\right)$. Certains commentateurs ont vu dans cette pièce une critique de la suprématie de la raison d'État, mais l'enjeu politique reste au second plan et est obscurci par la disposition. Il n'est pas question du mariage avant qu'un soldat n'en parle à Lélius et c'est seulement à cette occasion que Massinisse rappelle la promesse faite par Asdrubal. Surtout, le conflit des loyautés n'est jamais

(comme je pense) d'avoir par cette voie trouvé moyen d'assouvir sa luxure, et satisfaire aux prières de Sophonisbe. »

"TRISSINO, op. cit., v. 545 (« cortese ») et v. 549 (« liberal»); la promesse est ainsi formulée, v. 538-544: «e par maggior chiarezza la man destra / toccar vi voglio. E or per questa giuro, / e per quel Dio che m'ha dato favore / a racquistare il mio paterno impero, / che servato vi fia quel che prometto, / e non andrete in forza de' Romani / mentre che sarà vita in queste membra. » Mellin de Saint-Gelais traduit (op. cit., p. 263-4) : «Et pour plus grande sûreté je vous veux donner ma foi et la jurer en votre main, avec le Dieu qui $\mathrm{m}^{\prime} \mathrm{a}$ donné faveur au recouvrement de mon royaume, que vous n'irez en puissance d'aucun Romain tant que la vie me soutiendra. »C. DELMAS montre combien cette tragédie renaissante prend les accents du roman de chevalerie au mépris de toute réalité historique (op. cit., p. 457-458).

"TRISSINO, op. cit., v. 1418-1422. Mellin traduit (op. cit., p. 280) : "Mais bien vous veux-je supplier de n'être point mal content si je cherche d'acquitter ma parole et ma foi, laquelle avant qu'y bien penser j'ai obligée un peu trop soudainement ».

13 TRISSINO, op. cit., v. 1585-1593. Mellin, op. cit., p. 284 : "volontiers il vous eût tenu sa première promesse, mais puisqu'un autre plus puissant lui en a ôté le moyen, à tout le moins vous tient-il sa seconde. C'est que si vous voulez, vous ne tomberez point vivante en la puissance des Romains, vous conseillant en cet endroit acte digne de noble sang, dont vous êtes issue. »

${ }^{14}$ La scène est chez TITE-LIVE (XXX-12) et chez APPIEN (VIII-27). 
clairement exprimé. Comme le dit Gillian Sharman, Trissino s'intéresse plus aux effets qu'aux causes ${ }^{15}$.

De fait, le propos de La Carthaginoise, ou La Liberté est plus clair que celui de la Sophonisbe italienne. Il prend la forme d'une démonstration sans détour d'un principe simple, celui de la nécessaire fidélité au souverain légitime. Cela tient en particulier au fait que Montchrestien ne reprend pas les éléments de l'histoire d'Appien : la question d'une fidélité première à Sophonisbe ou à Carthage se trouve ainsi évacuée. Grâce aux Romains, Massinisse reprend possession de son royaume, usurpé par le tyran Syphax. En entrant dans la ville, le roi Numide rend grâce aux Dieux de punir ce dernier :

Ce traître usurpateur, ce meschant desloyal

Qui minutoit ma mort dans mon Throne royal

C'est montrer clairement que les âmes fidelles

Ont toujours pour abri vos faveurs paternelles'.

Plus loin, Scipion justifiera aussi cette destitution : "Car à nul n'appartient le sacré nom de Roy / Qui d'une perfidie ait entaché sa foy ${ }^{17}$. $\gg$ Le mariage n'est qu'une conséquence funeste de passions mal contrôlées, l'effet des charmes de Sophonisbe, que Syphax puis Scipion décrivent comme une sorcière (acte IV). C'est un ensorcellement bien plus qu'une trahison et la perfidie est essentiellement féminine.

Pour le rendre à la raison, le général romain adresse à Massinisse un discours aux accents néo-stoïciens ; il lui rappelle qu'il faut triompher de soi pour mériter d'être roi et que seule la vertu lui apportera la gloire. Massinisse reconnaît immédiatement son erreur : la fidélité au souverain doit être entière :

\section{Empereur redoutable, en te donnant ma foy \\ Je ne me réservay nulle chose de moy, \\ Sinon le seul désir de te rendre service : \\ Et bien, si tu le veux Sophonisbe perisse ; \\ Mais de grace permets que je puisse acquitter \\ Le solennel serment qu'elle m'a fait prester"s.}

Il lui enverra donc le poison en lui faisant dire qu'il a «faussé sa foy pour [lui] garder la foy ${ }^{19}$ », la promesse de lui ôter la vie pour lui épargner le triomphe venant contredire le serment conjugal. Mais la contradiction des fidélités se réduit à n'être qu'un paradoxe élégant qui vient souligner le double oxymore d'une reine qui meurt le jour de ses noces et d'un roi fidèle et parjure. La question de la fidélité politique, parce qu'elle prime sur toute autre, est en fait détachée, dégagée de celle d'une fidélité privée et issue de passions néfastes. Le propos s'inscrit d'ailleurs parfaitement dans la logique d'un huguenot fidèle au roi Henri IV et qui allait donner à l'État son premier traité d'économie politique. Il y a bien une douleur de Massinisse qui s'exprime dans une longue plainte au début de l'acte $V$, mais pas de déchirement à proprement parler : La Carthaginoise est l'histoire de l'écart passionnel commis par roi qui, parce qu'il doit sa souveraineté à ses alliés, leur est entièrement

\footnotetext{
${ }^{15}$ TRISSINO, Sophonisba, trad. et éd. Gillian Sharman, The Edwin Mellen Press, 1997. L'interprétation politique est d'ailleurs fragilisé par le dédicataire de la pièce, le pape Léon $\mathrm{X}$ : celui préparait alors la guerre contre le duc d'Urbin pour mettre à sa place son neveu, Laurent de Médicis.

${ }^{16}$ MONTCHRESTIEN, op. cit., acte II, p. 127.

"Ibid., acte IV, p. 143.

Isid., acte IV, p. 147.

${ }^{19}$ Ibid., acte V, p. 154.
} 
fidèle. Dans cette tragédie humaniste ${ }^{20}$, la question de la fidélité est surtout mobilisée pour créer un héros vertueux et exemplaire. Comme chez Pétrarque, à qui Montchrestien reprend les caractères de ses personnages, les mouvements de la plainte de Massinisse et jusqu'à ses métaphores, la pièce est une variation du conflit de la vertu et des passions bien plus qu'une tragédie de la fidélité.

Avec Mairet, l'histoire de Sophonisbe devient une «tragédie exclusivement amoureuse $^{21} \gg$. La question de la foi en politique se trouve pratiquement évacuée pour répondre au Pyrame et Thisbé de Théophile sur le terrain de l'histoire. « Mairet substitue au sujet historique du suicide de la reine carthaginoise fidèle à sa patrie et hostile à Rome un autre sujet : celui de la mort des amants comme effet non plus d'une transcendance historique et politique mais de la fatalité de la passion ${ }^{22}$. » La volonté de construire une tragédie élégiaque conduit le dramaturge à gommer le conflit des fidélités ${ }^{23}$. En fait, la trahison patriotique ou politique n'est là que pour souligner la force de la passion amoureuse. La relégation au second plan de l'enjeu politique, voire son élimination, tient à deux éléments : la passion des deux amants et la mort de Syphax. La promesse de mariage antérieure à l'union avec Syphax est évoquée par deux fois (I, 1 et $\mathrm{IV}, 1)$ mais elle n'intervient pas de manière décisive dans les événements : Sophonisbe tombe amoureuse de Massinisse en le voyant sur le champ de bataille lors d'affrontements qui précèdent le débarquement des Romains en Afrique (IV, 1, v. 1035-1072). Leur hymen se détache ainsi de la question des alliances guerrières. L'arrière-plan politique et militaire est ainsi totalement absent du récit que fait la reine de son innamoramento. Invention de Mairet, la mort de Syphax au combat, a deux conséquences. Premièrement, puisque Sophonisbe aime déjà Massinisse, son mariage devient une fin et non plus seulement un moyen pour conserver sa dignité de reine et sa fidélité à Carthage ; c'est un mariage d'amour et non plus un mariage politique. Deuxièmement, avec la disparition $\mathrm{du}$ roi des Masaesyles, Mairet se débarrasse de la question de la légitimité du mariage. Enfin, notamment parce que Syphax n'effraie plus Scipion avec le risque d'un Massinisse faisant défection sous l'influence de Sophonisbe, l'ordre des Romains s'impose de l'extérieur et n'est plus que l'occasion du dénouement funeste. Aux yeux de Massinisse, l'exigence romaine est un acte de tyrannie et non une conséquence de la foi qui le lie à eux. Il dit ainsi à Lélius :

Cruels, qui sous le nom de la chose publique,

Usez impunément d'un pouvoir tyrannique;

Et qui pour témoigner que tout vous est permis,

Traitez vos Alliés comme vos ennemis".

De la même manière que la politique fonctionne comme l'instrument de la fatalité de l'Amour, le thème de la trahison n'est convoqué que pour approfondir le pathétique amoureux et exalter la toute puissance de la passion. Celle-ci est telle que les serments et fidélités disparaissent devant elle : la trahison est une conséquence de l'amour. On voit ainsi Syphax, qui a intercepté une lettre de Sophonisbe destinée à

\footnotetext{
${ }^{2}$ L'enjeu est de rappeller aux rois et aux hommes combien est incertaine leur félicité. L'ouverture de l'acte III va dans ce sens : Furie tout droit sortie des Enfers, Mégère vient jeter le poison de la discorde entre les alliés ; le fléau de la guerre ne lui suffit pas, et elle veut "mouvoir de plus intestins débats », « rabaisser le courage» (Ibid., acte III, p. 134) du vainqueur et le plonger dans le désespoir.

"Ces mots sont ceux de B. LOUVAT (op. cit., p. 57). Mon analyse reprend bien des conclusions de sa longue et très complète introduction à la pièce.

"Ibid., p. 83.

${ }^{2}$ Le choix de faire mourir Massinisse, après une longue plainte sur le corps de Sophonisbe en forme de monologue final, est sans doute la preuve la plus éclatante de cet infléchissement.

${ }^{2}$ MAIRET, op. cit., V, 2, v. 1439-1442.
} 
Massinisse, s'accuser d'aimer ceux qui le trahissent (I, 2, v. 196). Le monologue que prononce Sophonisbe pendant que la bataille fait rage est particulièrement révélateur à cet égard. Puisqu'elle souhaite par amour la victoire de Massinisse sur Syphax, elle se trouve réduite à "vouloir le salut de ceux qui [la] détruisent » (II, 1, v. 352). Annonçant l'oxymore du vainqueur vaincu, le discours déploie celui de l'amie de l'ennemi ou de l'assiégée bienheureuse, à travers toute une série de pointes galantes reposant sur l'identification de la femme et de la ville. Elle s'adresse ainsi aux soldats :

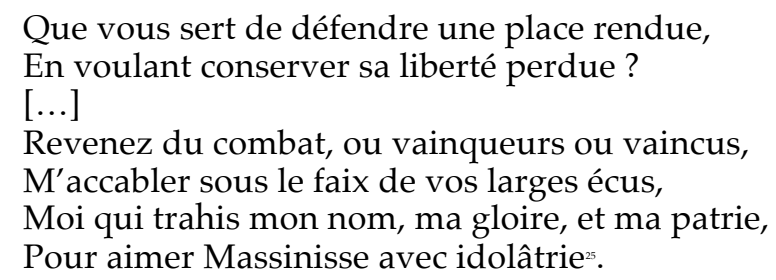

Le mouvement est le même lorsque Sophonisbe raconte à Massinisse l'origine de son amour pour lui : "De là je commençai de vendre mon pays, / Et de là dans mon cœur les miens furent trahis » (IV, 1, v. 1065-1066). Le contexte politique est à ce point gommé qu'on ne sait pas vraiment qui désigne "les miens », les Numides ou les Carthaginois.

\section{Montreux, Marston et Corneille : destructions et constructions de la fidélité}

Avec Montreux, Marston et Corneille, la trahison (ou son refus) n'est plus seulement l'un des éléments de l'élaboration du tragique : elle devient le principe qui ordonne les actions et les discours. La question de la fidélité (ou la tentation de la trahison) ne se résout plus par l'affirmation d'une morale incontestée mais régit les relations entre les personnages, leurs conflits et leurs actions, se déploie dans leurs discours qui se font l'écho de controverses du temps. Son articulation avec la justice, avec les conditions d'énonciation de la promesse, avec les questions de l'identité ou du langage permet d'en explorer les contradictions et les enjeux.

La Sophonisbe de Nicolas de Montreux est entièrement consacrée à une réflexion sur la foi en politique. Pour ce faire, le poète, qui s'appuie sur la seule version $\mathrm{d}^{\prime}$ Appien, a éliminé du sujet toute dimension amoureuse ${ }^{26}$. La pièce commence après l'union de Massinisse et de Sophonisbe, avec l'affrontement de Syphax et de Scipion qui lui demande raison de sa trahison. Dans le conflit qui l'oppose aux Romains, jamais Massinisse n'invoque l'amour qu'il porte à la reine de Numidie et, surtout, jamais le texte ne met en présence les deux époux. La dédicace de la pièce indique elle aussi que le poème a avant tout un enjeu politique. La tragédie est dédiée à Monsieur de Montgommery, avec ces mots : "Ceci est une liberté pour une autre: feu Monsieur de Lorges vostre frere et vous me fistes libre de prison, et je vous offre la Sophonisbe libre de servitude ${ }^{27}$. » Si l'on se souvient que Montreux a longtemps été sous la protection du duc de Mercoeur, cousin des Guises et dernier grand prince ligueur à signer la paix avec Henri $\mathrm{IV}^{2 s}$, on peut s'étonner de le voir choisir l'un des chefs huguenots comme dédicataire. Pendant les guerres de religion, Montreux avait

\footnotetext{
sIbid., II, 1, v. 383-384 et 389-392.

${ }^{2}$ C. Delmas et B. Louvat soulignent tous les deux cette particularité de la pièce.

"Nicolas de MONTREUX, op. cit., p. 35.

" À Nantes en 1598. La paix signée avec le duc de Mercoeur, gouverneur de Bretagne, ouvre justement la voie de l'Édit de Nantes. Pour des données biographiques sur Nicolas de Montreux, dont le pseudonyme était Ollenix du Mont-Sacré, voir J. MATHOREZ, Le poète Olényx du Mont-Sacré, Bibliothécaire du duc de Mercoeur (1561-1610), Paris, Henri Leclerc, 1912 et l'introduction de Donald Stone.
} 
embrassé assez tôt la cause des Ligueurs ; il s'était retrouvé emprisonné, avec ses biens confisqués, et c'est certainement à cette occasion que les frères Montgommery l'avaient sorti de ce mauvais pas. Cet événement a nécessairement eu lieu avant 1591, date de la mort du comte de Lorges. Le poète se réfugia alors à Nantes, chez les Mercoeur qui en font leur bibliothécaire. En 1601, une fois le duc de Mercoeur parti combattre les Turcs en Hongrie, Ollenix du Mont-Sacré se retrouvait sans protecteur officiel et Montgommery était sans doute une marche vers la cour. Reste que la dédicace de $\mathrm{La}$ Sophonisbe manifeste une reconnaissance pour des hommes qui appartiennent au camp opposé ; le choix du dédicataire témoigne que Montreux connaît les épreuves rencontrées par la fidélité en temps de guerres civiles, et aussi après la paix. Sa tragédie porte très nettement la marque de cette expérience.

Le premier acte pose la question du transfuge, du changement de camp. Après une longue plainte de Syphax qui développe le topos de l'inconstance du sort des grands, Scipion et Lélius viennent lui rappeler qu'un roi doit savoir garder sa foi et lui rappellent que les dieux «sont vangeurs du brizement des lois, / Punisseurs éternels de la foy parjurée» (v. 252-253). Face aux accusations de "meschant infidelle, / Trahissant l'amitié de nature immortelle»(v. 261-262), Siphax a une défense mouvante. Il rejette la faute sur Sophonisbe, qui « ravit par ses yeux [sa] chère liberté » (v. 376). Mais il invoque aussi un lien de fidélité naturel, culturel et politique, voire économique, qui le liait aux Carthaginois dont il s'était à tort éloigné :

J'ay fait ce que j'ai deu en prenant la deffance

De ceux de qui j'avois recherché l'alliance,

Qui m'estoyent alliez d'air, de sang et de meurs

Et dont j'avois receu mille sacrez honneurs.

J'ay donc fait mon devoir".

À quoi Lélius répond qu'une fois une promesse donnée, il faut la garder et qu'une deuxième promesse ne vient pas annuler la première. Siphax fait alors valoir que le mariage, «lien tressaint » qui doit unir deux volontés, lui imposait cette trahison qui n'en est pas une : puisqu'il devait être fidèle à deux partis, " pour changer de party ce n'est estre infidelle» (v. 413). Les Romains opposent aux difficultés de circonstances du Numide un principe immuable : on ne peut avoir qu'une foi ; elle fonde la légitimité du roi et la justice, «Car la loy n'est plus loy qui fait mourir la foy, / Puisque la seule foy est gage de la loy » (v. 403-404).

Avec le troisième acte, $c^{\prime}$ est la question de la rébellion qui se pose : un serment doit-il être respecté au prix de la révolte ou bien doit-on se parjurer pour obéir au souverain auquel on a fait allégeance? C'est bien la situation dans laquelle se trouve Massinisse, désormais marié à Sophonisbe, à qui il a promis la liberté mais que Rome réclame comme sienne. Indigné, offensé par son allié qui ne reconnaît pas l'obligation qu'il a envers lui pour ses victoires, le roi Numide envisage de prendre les armes contre Rome. Il a avec lui Gelosses et contre lui Misipsa, son fils. Gelosses argue d'une forme de fidélité à l'Afrique contre les étrangers que sont les Romains, dans laquelle on serait tenté de reconnaître la position de certains seigneurs face au pouvoir royal :

Chassons ces estrangers, et que nostre patrie

Soit exempte ce jour de leur vive furie

Recognoissons nos loix, nostre pays, nos citez,

Et deffendons encor nos cheres libertez,

Nos temples nos palais, et de nos premiers peres,

trespassez pour le pays, les tombes mortuaires ${ }^{30}$.

"MONTREUX, op. cit., v. 349-353.

${ }^{30}$ Ibid., v. 1413-1418. 
Massinisse refuse de renier sa foi, notamment parce qu'elle a été donnée à un être en position de faiblesse. La position de Misipsa fait de lui un pragmatique quelque peu machiavélien. Il recommande la prudence: face à la défaite assurée contre les Romains, il suggère de ne pas "garder la promesse qui fait / Un estat misérable en son cruel effait » (v. 1559-1560). En revanche, Sophonisbe ne pourra rien faire contre eux (celui qui est asservi ne peut contraindre au devoir). Enfin, il affirme que le serment impossible est toujours révocable. Mais il invoque aussi des principes moraux pour régler le conflit des fidélités : la foi publique doit primer sur la foi privée, la foi ancienne sur la nouvelle, le bien public sur la foi donnée à l'individu :

Une petite foy qui brise la publique,

Une promesse neuve empeschant une antique

Ne sont foy ni serment, puisqu'elles font périr

Celles que nous devons pour divines cherir"

Enfin, de manière plus discrète qu'avec ces deux grandes disputes mais tout au long de la pièce, la question de la fidélité rencontre celle de l'équité, mise en danger par la guerre. Plusieurs fois l'idée que la foi est celle du plus fort se trouve exprimée. Elle l'est d'abord par Siphax qui parle du vainqueur « qui force par le fer et le droit et la loy, / Et rasseure ses ans par la mort de la foy » (v. 207-208). Les deux rois Numides expriment leurs doutes quant au bien-fondé des intentions des Romains ambitieux et orgueilleux. Ailleurs, Sophonisbe compare sa servitude à celle de Massinisse, placé sous l'autorité des Romains : "la seule force regne et non pas l'equité » (v. 873). Au moment de mourir, elle fera le même constat d'une guerre qui détruit le droit, justifiant par là un Massinisse qui vient de se reprocher son absence de loyauté : il est parvenu à "garder immuable [sa] foy » en lui permettant de rester libre grâce au poison. Enfin, les stichomythies qui suivent les tirades de l'acte III et en reprennent les principales oppositions explorent dans leurs méandres les impasses de la foi donnée quand le rapport de forces est désavantageux.

On peut se demander si la dédicace livre une clef de la pièce: Montreux s'assimilant à Sophonisbe, Massinisse pourrait être identifié à Montgommery, Siphax à Mercoeur et Scipion à Henri IV. Le schéma fonctionne assez bien dans l'ensemble, l'héroïsme de Massinisse venant confirmer l'éloge du dédicataire dont on cherche à faire un protecteur et les deux Numides occupant la place des seigneurs dont la souveraineté dépend désormais entièrement du roi. Il y a cependant des limites évidentes à cette transposition : le discours sur la dignité et le pouvoir royaux concerne les deux rois Numides, le roi de France devient alors une puissance étrangère et Mercoeur, à la louange duquel Montreux écrira la chronique de ses exploits contre les Turcs, prend la place du traître Siphax. Mais il convient de remarquer que Siphax est bien moins "traître » que chez un Tite-Live ou un Montchrestien. Les raisons qu'il avance à l'acte I ont une certaine légitimité en ce qu'elles soulignent la complexité du lien de fidélité et le fait que la foi n'est pas toujours une. En outre, la pièce fait assez clairement entendre l'idée qu'en temps de guerre, et particulièrement en temps de guerre civile (rappelons que Massinisse et Syphax sont tous deux Numides), le respect des serments est parfois difficile voire impossible à tenir. Il est malaisé de sortir de cette mise à l'épreuve des fidélités sans faire d'accroc à la vertu et l'on peut voir des héros qui deviennent parjures et traîtres sans pour autant cesser d'être héros. Ainsi à l'acte V, Massinisse s'accuse longuement de sa déloyauté (v. 2302 et suiv.). Les guerres intestines conduisent à des conflits des fidélités insolubles, sinon dans la mort - celle de Sophonisbe permettant ici au roi

"Ibid., v. 1565-1568. 
Numide de garder sa foi doublement et contradictoirement donnée. Finalement, la tragédie de Montreux insiste sur l'ambivalence des positions occupées par les personnages, la complexité de la situation et le rôle des circonstances - ce qui était aussi une manière pour Ollenix de Mont-Sacré de plaider sa cause auprès du chef huguenot.

Les oppositions sont beaucoup plus tranchées dans la pièce de Marston. The Wonder of Woman, or the Tragedie of Sophonisba, publiée en 1606 et jouée par la troupe d'enfants du théâtre des Blackfriars ${ }^{32}$, se distingue du reste de la production dramatique de son auteur: plus sérieuse, elle est sans trace du cynisme ou de la satire qui caractérisaient les pièces précédentes ${ }^{33}$. Les caractères et l'intrigue sont en effet composés de sorte à opposer clairement les fidèles d'une part (Sophonisbe, Massinisse et Gelosso, un sénateur de Carthage) et les traîtres d'autre part (Syphax, Hasdrubal, Hannon et Carthalon, sénateurs et capitaines de Carthage, et Zanthia, chambrière de la reine). La différence avec les autres réécritures, qui tient en particulier dans la concentration des événements, le montre de manière éclatante. L'action se situe en Afrique et s'ouvre sur la célébration des noces de Sophonisbe et de Massinisse, qui scellent l'alliance de celui-ci avec Carthage. Humilié et dépité, Syphax décide de rejoindre Scipion pour se venger et donc de trahir les Carthaginois dont il était aussi l'allié :

The winged vengeance of incensed Jove

Upon this Carthage : for Syphax her flies off

From all allegeance, from all love or service

His (now freed) scepter one did yeeld this Cittie".

Au moment du coucher solennel des deux époux, Carthalon apporte la nouvelle : grâce à l'appui de Syphax, les troupes de Scipion sont en train de prendre le dessus. Sans même consommer le mariage, Massinisse part au combat pour défendre Carthage. Les sénateurs carthaginois, à l'exception de Gelosso, décident alors de rompre les engagements qui les lient à Massinisse pour donner Sophonisbe à Syphax et regagner l'alliance de ce dernier (II, 1). Ils vont jusqu'à fomenter un complot contre le roi des Massyles, mais la tentative d'assassinat par Giscon l'empoisonneur échoue grâce à Gelosso (II, 2). Toujours sur le champ de bataille, blessé, attaqué par les troupes carthaginoises, Massinisse est alors contraint de passer à l'ennemi. La protection de Scipion devenant la condition de sa survie, il est ainsi lavé de toute trahison. Les actes III et IV sont occupés par les tentatives de Syphax pour posséder Sophonisbe, tentatives qui s'échouent sur la fidélité et la vertu sans exemple de la reine. Au dénouement, quand Syphax s'apprête à aller combattre l'armée de Massinisse, le spectre d'Hasdrubal lui raconte son sort pitoyable et met en garde les traîtres contre les châtiments des dieux: voyant dans sa trahison la raison de la défaite, le peuple l'a privé de sépulture. Il n'y a là qu'une juste infortune $(\mathrm{V}, 1, \mathrm{v} .46$, «justly infortunate ») :

\footnotetext{
"Les troupes élisabéthaines se partagent deux types de scènes dans la capitale: les théâtres dits «publics », comme celui du Globe, où jouent les troupes professionnelles d'adultes, et les théâtres dits «privés » où jouent les troupes d'enfants : ce sont des adolescents très bons danseurs, musiciens et chanteurs (la pièce de Marston ménage d'ailleurs beaucoup de place à la musique).

${ }^{3} C^{\prime}$ est la dernière pièce de Marston. Après quelques écrits de cour, il quittera la scène littéraire pour devenir prêtre en 1609. Voir notamment Peter URE, «John Marston's Sophonisba : a Reconsideration », 1949, repris dans Elizabethan and Jacobean Drama, Liverpool, Liverpool University Press, 1974, p. 75-92 ; John Scott Colley, John Marston's Theatrical Drama, Salzburg, 1974 ; George L. Geckle, John Marston's Drama. Themes, Images, Sources, Londres-Toronto, Associated University Presses, 1980, en particulier le ch. 10 : «The Woes of Vows in Sophonisba».

"MARSTON, op. cit., I, 2, v. 49-52.
} 
Mortals O feare to sleight

Your Gods and vowes: Joves arme is of dread mights.

Quant aux caractères, comme le fait remarquer Jacqueline Pelorson, ils font l'objet d'un travail d'abstraction : les personnages sont tout entiers régis par un principe ou un affect. Syphax est gouverné par le désir et le plaisir des sens. Massinisse incarne la justice et Sophonisbe la fidélité. On le voit, la pièce sépare clairement les vertueux des coupables. Mais cette simplicité n'est qu'apparente et le texte travaille la notion de trahison de trois manières : d'abord avec le débat entre Gelosso et les sénateurs, ensuite par la place importante donnée à la figure du traître, enfin par l'articulation de la fidélité au stoïcisme et aux questions du langage et de l'identité.

La première scène de l'acte II, où l'on voit les Sénateurs briser leur foi donnée à Massinisse pour gagner Syphax, pose les termes d'un débat sur les rapports entre la morale et la politique. Marston mobilise la pensée de Machiavel, mais la séquence s'appuie plus directement sur le premier chapitre du Livre III des Essais, « De l'utile et de l'honnête ", véritable méditation sur la trahison ${ }^{36}$. Le dramaturge cependant en radicalise les oppositions en distribuant les considérations entre les vertueux et les traîtres, et sa conclusion ne laisse rien subsister des nuances ou des exceptions envisagées par Montaigne. Hannon et surtout Carthalon se font ainsi les défenseurs d'un machiavélisme presque caricatural : "Profit, and Honesty, are one in state » (II, 1, v. 14) ${ }^{37}$; le succès fait oublier les moyens employés (v. 33-34) et le bien de l'État ne doit pas s'embarrasser de scrupules moraux (v. 67-68). Le discours de Carthalon, du v. 55 au v. 68, décalque pratiquement mot à mot les morceaux suivants du chapitre «De l'utile et de l'honeste »:

Il n'y a rien d'inutile en nature, non pas l'inutilité mesme ; [...] Nostre estre est simenté de qualitez maladives [...]. De mesme, en toute police : il y a des offices necessaires, non seulement abjects, mais encores vicieux : Les vices y trouvent leur rang et s'employent à la cousture de nostre liaison, comme les venins à la conservation de nostre santé. [...] Le bien public requiert qu'on trahisse, et qu'on mente, et qu'on massacre ; [...] Le Prince, quand une urgente circonstance, et quelque impetueux et inopiné accident, du besoing de son estat luy fait gauchir sa parolle et sa foy, ou autrement le jette hors de son devoir ordinaire, doibt attribuer cette necessité à un coup de la verge divine : Vice n'est-ce pas, car il a quitté sa raison, à une plus universelle et puissante raison, mais certes c'est malheurs.

À quoi Gelosso répond que la trahison est affaire de bourreaux, qu'il préfère conserver son honneur, que celui qui refuse de trahir un homme pour sa patrie refusera de trahir sa patrie pour un homme et qu'enfin, celui qui est infidèle à luimême ne peut être blâmé s'il l'est ensuite envers le prince ${ }^{3 .}$. Pour lui, foi privée et foi publique se fondent réciproquement, et il n'y a pas de différence entre la fidélité (ou la trahison) à un particulier et la fidélité (ou la trahison) patriotique ; la deuxième ne permet pas de se dégager de la première. Alors que les Sénateurs revendiquent ce

\footnotetext{
${ }^{35}$ Ibid., V, 1, v. 61-62.

${ }^{36}$ Les Essais de Montaigne étaient disponibles depuis 1603 dans la traduction de John Florio et étaient lus dans les milieux littéraires londoniens.

${ }^{37}$ Le vers transpose le titre de l'essai : «Of Profit, and Honesty ».

${ }^{38}$ Montaigne, Essais, III, 1, éd. Jean Balsamo, Michel Magnien et Catherine Magnien-Simonin, Paris, Gallimard, « Bibliothèque de la Pléiade », 2007, p. 830 et p. 840.

${ }^{39}$ " And he thats faithlesses to his proper selfe, / May be excusd if he breake faith with princes » (II, 1, v. 84-85) ; «Qui est infidelle à soy-mesme, l'est excusablement à son maistre. » (Ibid., p. 32)
} 
que Montaigne constate ou autorise dans certaines circonstances ${ }^{40}$, Gelosso, à qui le dénouement donnera raison, érige en principe ce que Montaigne revendique pour lui-même ${ }^{41}$.

Le propos du fidèle sénateur va se trouver confirmé indirectement par le personnage de Syphax. Marston en fait un tyran luxurieux et violent; il souligne ainsi chez le traître une continuité entre le privé et le public't Sa tyrannie apparaît dès l'ouverture de la pièce lorsqu'il affirme: "Passion is Reason when it speakes from Might» (I, 1, v. 77). C'est parce qu'il est esclave de ses sens que Syphax a trahi Carthage. La même idée est présente implicitement dans la question que pose Massinisse à Giscon, une fois son projet meurtrier découvert : "Art poore ? (II, 2, v. 44). Ce sont les hommes asservis à leurs appétits, incapables de maîtrise de soi qui font les traîtres. Aussi les voit-on s'enfoncer toujours plus avant dans la trahison. Lorsque Syphax blâme les charmes lascifs de Sophonisbe pour expliquer à Scipion la rupture de l'alliance, il n'a jamais été aussi traître puisque la Carthaginoise de Marston est la plus pure des Sophonisbe. L'épisode magique de l'acte IV va aussi dans ce sens. Le roi Numide croit posséder enfin la reine alors qu'il s'unit à l'horrible sorcière Erichto, qu'il avait lui-même invoquée ; or, dans la poésie latine chrétienne, l'on trouve parfois Judas en compagnie de la sorcière de la Pharsale ${ }^{\text {as. }}$.

Face à cette figure du traître par excellence se dresse celle de Sophonisbe qui lui est symétriquement opposée. Chaste, tempérante, maîtresse d'elle-même et douée d'une force d'âme remarquable, elle est ainsi très différente de la source latine qui insistait sur son charme sensuel et son pouvoir de séduction. Modèle de la fidélité absolue et de perfection vertueuse, Marston en fait le centre dramatique de sa pièce et la figure héroïque agissante. C'est elle qui prend l'initiative du suicide à l'acte $\mathrm{V}$ et qui offre sa mort pour sortir Massinisse du piège de la perfidie créé par les deux serments contradictoires :

My death

Giv's helpe to all : From Rome so rest we free,

So brought to Scipio, faith is kept in thee".

La grandeur de cette "merveille des femmes » apparaît en particulier dans le conflit qui l'oppose à Syphax: bien loin de reconduire une représentation allégorique du combat entre Fidélité et Traîtrise, celui-ci se trouve transposé dans une action dramatique, spectaculaire et physique. Pour échapper à Syphax et préserver le serment qui la lie à Massinisse, Sophonisbe a d'abord recours à la ruse : elle réclame une heure pour un sacrifice, puis elle s'enfuit par un souterrain en plaçant dans le lit conjugal un esclave endormi pour tromper Syphax. Mais elle est trahie par Zanthia et Syphax la surprend à la sortie du souterrain alors qu'elle se repose. C'est à ce moment qu'a lieu le miracle : sa vertu devient une véritable force agissante et Syphax « émerveillé » («amazde») renonce à la violer. Or, ce «pouvoir / Qui [la] fait si

\footnotetext{
${ }^{4}$ On peut être traître et rompre ses serments avec le traître lui-même, quand l'utilité publique exige la mort d'un tyran (mais non pour augmenter le revenu public), ou bien lorsqu'on a promis une chose " méchante et inique de soi ».

"Ibid., p. 29 : «Tendre négociateur et novice, qui aime mieux à faillir à l'affaire qu'à moi !". Ibid., p. 43 : «toutes choses ne sont pas loisibles à un homme de bien pour le service de son roi ni de la cause générale et des lois. »

"Au début de l'acte III, la didascalie précise qu'il entre en scène en traînant Sophonisbe par les cheveux, qu'il tient enroulés autour de sa dague ; l'idée du viol attise son désir et il envisagera même de satisfaire son appétit sexuel avec Zanthia en attendant sa promise.

"Paul-Augustin DEPROOST, "La figure de Judas dans la poésie latine chrétienne ", Figures du traître. Les représentations de la trahison dans l'imaginaire des lettres européennes et des cultures occidentales, études réunies par Jean-Jacques Pollet et Jacques Sys, Arras, Artois Presses Université, 2007.

"MARSTON, op. cit., V, 3, v. 85-87.
} 
glorieuse » («that power / By which thou art thus glorious », IV, 1, v. 79-80) se manifeste justement par la réaffirmation de sa fidélité à Massinisse :

I speake all frightles, know I live or die

To Massinissa, nor the force of fate

Shall make me leave his love, or slake thy hate.

I will speak no mores.

Ce sont ces mots qui tiennent Syphax à distance, préservant ainsi sa foi et sa chasteté. On voit par là que pour Marston la fidélité est au principe de la vertu et qu'elle en fonde l'efficacité.

Ce n'est pas le seul rôle que lui donne Sophonisbe : elle est aussi garante du langage et de l'identité. En effet, lorsqu'elle apprend le contenu du décret infidèle, reprenant les mots de Montaigne au chapitre $9 \mathrm{du}$ premier livre des Essais («Des Menteurs »), elle dit aux Sénateurs :

We all have sworne good Massinissa faith,

Speach makes us men, and thers no other bond

Twixt man and man, but words : O equall Gods

Make us once know the consequence of vowes".

Par deux fois, elle dit sa méfiance du protocole et de la rhétorique, c'est-à-dire des formes, plus ou moins figurées, qui sont privées de sens parce qu'elles ne renvoient plus à aucune réalité (I, 2, v. 6-28; IV, 1, v. 21-27) ${ }^{\star}$. On le voit, cette inquiétude, particulièrement aiguë dans l'Europe du seuil de la modernité, est ici indissociablement liée à la question de la fidélité. Le langage ne peut rester un instrument de vérité et un lien entre les hommes que si l'on agit conformément à ce que l'on a dit, que si les actes répondent aux serments. Le chaos du dialogue entre les traîtres, après que le plan a échoué, le prouve clairement (II, 3, v. 94-113). Et comme le langage est ce qui fait de nous des hommes, la fidélité est aussi ce qui fonde l'identité. Gelosso l'avait dit quelques vers auparavant: "Our vow, our faith, our oath, why th'are our selves » (II, 1, v. 83), notre foi et nos serments sont nous-mêmes. Cette idée, la pièce de Marston la met en scène de deux manières particulièrement frappantes. L'oxymore qu'est Sophonisbe en est la première illustration: par la rupture de la foi, les hommes ont fait d'elle " a maide, a widow, yet a hapless wife » (II, 1 , v. 154 ; «Épouse infortunée, et vierge, et veuve aussi »), c'est-à-dire un impossible, quelque chose qui n'a pas de sens. La deuxième preuve tient dans la brève folie de Massinisse, unique moment de frénésie chez ce modèle de conduite stoïcienne, qui survient lorsqu'il prend conscience de la contradiction entre ses deux promesses. Il exprime alors sa douleur dans une syntaxe brisée qui peine à faire sens : «Right which way / Runne mad impossible distraction» (V, 3, v. 80-81; "C'est vrai. / Que faire ? Je suis fou! Nulle issue. O démence!»). S'esquisse alors l'idée que l'identité tient à la fidélité, qu'être infidèle à autrui c'est être infidèle à soi-même.

Plus d'un demi-siècle plus tard, cette idée devient évidente avec une autre Sophonisbe, celle de Corneille ${ }^{\star s}$. Mais ce dernier va plus loin : il apparaît alors

\footnotetext{
"Ibid., IV, 1, v. 65-68; «Je te le dis / Et n'en ai point peur, je vivrai ou mourrai / Fidèle à Massinisse. Te haïr et l'aimer, / Nulle fatalité ne m'en peut détourner. / J'ai dit. »

"Ibid., II, 1, v. 117-120 ; "Tous au preux Massinisse nous avons juré foi ; / La parole fait l'homme et, d'homme à homme, il n'est / De liens que les mots. O vous, dieux équitables, / Faites-nous bien sentir que nos vœux nous engagent. »

"Il est intéressant de remarquer que Marston s'éloigne ici de nouveau de Tite-Live, qui faisait de Sophonisbe une excellente oratrice.

* Je ne peux qu'évoquer trop rapidement cette très belle pièce, dans laquelle Doubrovsky voit d'ailleurs l'un des chefs-d'œuvre de Corneille; nous sommes un peu loin du XVI siècle et l'analyse en serait trop longue.
} 
clairement que la fidélité à soi fonde la fidélité tout court. Dans la réécriture cornélienne, Sophonisbe n'accepte de se marier que parce qu'elle pense qu'elle peut regagner Massinisse à la cause de Carthage, qui est toute sa passion. Elle s'identifie à sa patrie et toutes ses fidélités sont subordonnées à celle-là. Ailleurs dans le texte, la forme réfléchie $\mathrm{du}$ verbe "se trahir», employée au sujet d'un sentiment, est précisément équivalente à la rupture d'un serment. Mais Corneille ajoute une autre condition de possibilité à la fidélité, qui est le pouvoir, ou la liberté. Une fois Syphax captif, Sophonisbe ne voit plus aucun lien de fidélité qui les unirait. Certes, et le dramaturge le rappelle pour se défendre des attaques sur le double mariage, dans la loi romaine la captivité brise le nœud conjugal ; mais le texte y insiste trop pour ne pas y accorder plus d'importance. Eryxe, reine de Gétulie et rivale de Sophonisbe, le dit aussi à Massinisse : sa foi jurée à Sophonisbe est déjà une trahison puisque les Romains ne lui laisseront pas ce pouvoir. Les explications de Syphax à Scipion prennent ainsi un tout autre sens que dans les autres versions de l'histoire: "Lorsque je vous aimai j'étais maître de moi, / Et tant que je le fus je vous gardai ma foi » (IV, 2, v. 1187-1188). Le dénouement est peut-être plus éclairant encore à cet égard. Sophonisbe ne voit plus dans le poison une marque de la fidélité de Massinisse : d'abord parce qu'elle avait le sien propre, et surtout parce qu'il montre par là sa soumission à Rome. Lui-même le sait puisqu'il reconnaît ainsi " dégager sa foi » (V, 2, v. 1598). Surgit alors un paradoxe de la fidélité, puisqu'elle se fonde dans la liberté (je dois être libre pour me lier). Mais le paradoxe se résout dans la dualité du serment de fidélité (je jure fidélité à l'autre et à moi-même). Une vraie difficulté se dégage cependant de la pièce de Corneille, celle qui réside dans l'affirmation implicite que le seul moi qui s'appartient, et qui donc peut promettre, est le moi royal ${ }^{\natural}$.

Par la place de la trahison dans l'histoire de Sophonisbe, ce sujet s'est révélé particulièrement intéressant pour les dramaturges des $\mathrm{XVI}^{\circ}$ et $\mathrm{XVII}{ }^{\circ}$ siècles. Tous $\mathrm{y}$ ont puisé des situations tragiques grâce aux alliances scellées par les mariages et au conflit des fidélités. Certains d'entre eux, grâce à des héros à la fois fidèles et parjures $^{50}$, et à la multiplicité des épreuves de la fidélité, y ont aussi trouvé l'occasion de représenter l'expérience des guerres civiles, ou de s'interroger sur les enjeux de la foi donnée. Si aucune des tragédies n'adopte un point de vue cynique ni ne remet en cause le principe de la fidélité, celles de Montreux et de Marston portent la marque d'une évidence qui s'est perdue, chez le premier avec la nécessaire prise en compte des circonstances et des multiples aspects de la fidélité, chez le second avec le repli quelque peu mélancolique sur la forteresse intérieure du stoïcien. Si l'on considère ces textes dans la longue durée, on voit se dessiner le passage d'un ordre dans lequel on se donne tout entier sans jamais questionner la loyauté, à un autre, plus chaotique, où les fidélités (notamment celle qui relève du particulier et celle qui relève de l'État) ne s'ordonnent plus en une seule en se rangeant hiérarchiquement. L'une des réponses apportées par les pièces à ce désordre est celle de la fidélité à soi - à quoi fera écho, bien plus tard, La Conspiration de Nizan : le traître est le jeune homme au nom étrange, sans père, sans réelle appartenance, sans identité.

\footnotetext{
* La difficulté tombe si l'on fait l'hypothèse avec Hélène Merlin-Kajman que Corneille s'est concentré sur la sphère royale parce que «seuls les personnages royaux pouvaient figurer un moi souverain sans cesser d'être vraisemblables et sans se faire remarquer comme libertins » (L'absolutisme dans les lettres et la théorie des deux corps. Passions et politique, Paris, Champion, 2000, p. 226).

* Il est particulièrement remarquable à cet égard qu'aucun des personnages ne se prête au jeu de réversibilité du héros au traître et du traître au héros auquel se livre Borges dans Fictions («Thème du traître et du héros », "Trois versions de Judas »). Le héros Massinisse est aussi traître, et c'est cette tension qui débouche sur le tragique.
} 
